\title{
INFLUENCE OF THE MEDIUM UPON THE ORIENTATION OF SECONDARY TERRESTRIAL ROOTS
}

\section{RichaRd M. HolmaN}

In a recent paper ${ }^{1}$ I called attention to the inadequacy of the explanations put forward by earlier investigators for the striking difference in the behavior of primary roots which have been diverted from their normal position while growing in air or water, on the one hand, and earth, sand or other non-fluid medium, on the other hand. My experiments led to the conclusion that, after the primary root in air or water has flattened the primary geotropic curvature, a considerable resistance on the part of the medium to the advance of the root tip is a necessary condition for a subsequent complete curvature of the root. After the flattening of the primary curvature, the root in air grows straight ahead in an oblique position. Although the increasing length and weight of the root may result in its reaching the perpendicular through bending under its own weight, active reaction to the stimulus of gravity is generally restricted, after the completion of the autotropic flattening, to the extreme tip. This tip curvature, although varying in intensity, is maintained as long as the root is well supplied with water, is actively growing and has not attained an approximately perpendicular position. In such media as earth, sand, sawdust, and sphagnum, which offer more or less resistance to the root's advance, a root with such a curvature of the tip curves downward into the normal position in a curve whose radius is smaller the greater the resistance offered to the advance of the root. This downward curvature is apparently due to a passive depression of the root resulting from the non-symmetrical application (relative to the axis of the root) of the force opposing the advance of the root tip. These conclusions, arrived at as the result of a study of the behavior of the primary roots of Vicia faba, Lupinus albus, and Pisum sativum, naturally suggested the question, whether or not the secondary roots of these species showed any relation between geotropic behavior and medium such as exists in the case of primary roots.

1 The orientation of primary terrestrial roots with special reference to the medium in which they are grown. Amer. Journ. Bot. 3: 274-318. I916. 
In the course of the experiments upon the curvature of primary roots in different media, I had noted that the secondary roots arising from the upper side of a primary root, which had been planted horizontally in loose moist sawdust or just below the surface of very loose soil, showed little tendency to bend downward. They frequently bent so slowly that they emerged from the medium and grew obliquely

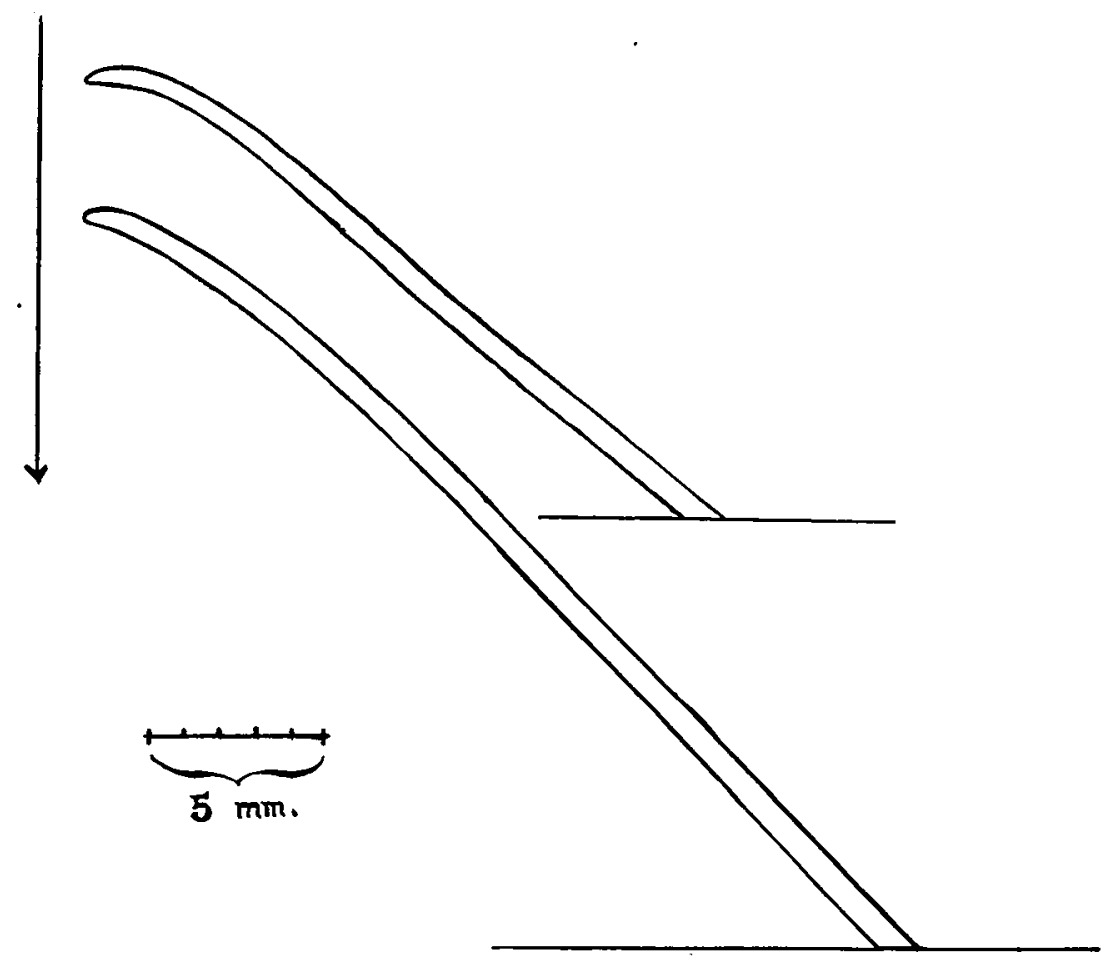

FIG. I. Secondary roots of Vicia faba, which arose from a primary root planted horizontally near the surface of fine moist soil. After emerging from the soil the roots grew upward into the air as shown in the figure.

upward into the moist air above the cultures for several centimeters. In such cases they never re-entered the medium from which they

2 Compare Sachs, Ueber das Wachsthum der Haupt- und Nebenwurzeln. Arb. Bot. Inst. Würzburg. I: 633. I874. Here Sachs reports the emergence of secondary roots from the soil when the mother root was growing perpendicularly. The secondary roots arose from the hypocotyl but those of which I speak had their origin I to $4 \mathrm{~cm}$. below the root base. 
had emerged. ${ }^{2}$ Figure I shows camera drawings of two such roots. Of these roots, the upper one had grown $2.2 \mathrm{~cm}$. after leaving the soil (the surface of which is represented by the horizontal lines) and the lower one $3.2 \mathrm{~cm}$.

Sachs $^{3}$ is the first author who reported any comparative observations of the behavior relative to gravity of secondary roots in different media. His principal observations were, Ist, that the limiting angle (Grenzwinkel) of secondary roots growing in air was greater than that of roots growing in water and greater in roots growing in the latter medium than in the case of secondary roots growing in earth, and, 2d, that, in air, the relatively acute curvatures resulting from displacement of the roots upward from the limiting angle or from subjecting them to a stimulus greater than gravity, by means of the centrifuge, were later flattened. Sachs employed principally seedlings of Vicia faba. Relative to the first of Sachs' observations, stated above, Czapek $^{4}$ reported that wetting of relatively dry sawdust cultures resulted in an increase, rather than a decrease, in the limiting angle of secondary roots growing in the sawdust. This result was just the opposite of what Sachs had reported when dry soil cultures were abundantly wetted. Both Czapek and Sachs stated however that the behavior of roots under the conditions mentioned was very inconstant. Sachs's own statement ${ }^{5}$ that secondary roots in air which are not frequently wetted lose their ability to bend downward suggests that the roots which showed a smaller limiting angle in air than in water may have behaved thus on account of too low humidity of the air surrounding them.

My own experiments with Vicia faba var. equina and var. major and with Lupinus albus and Pisum sativum disclosed a striking parallel in the behavior of primary roots and secondary roots of the first order. Vicia faba was used for most of the experiments because of the large number of secondary roots which it produces and the great vigor of growth which they display. (There are usually five rows of secondary roots in the case of Vicia faba, two rows in the case of Lupinus albus and three in the case of Pisum sativum.) The media used were air,

${ }^{3}$ L. c., 609 ff.

4 Czapek, Ueber die Richtungsursachen der Seitenwurzeln und einiger anderer plagiotroper Pflanzentheile. Sitzungsb. Akad. Wiss. Math. Naturw. (Wien) I04: I Abt: 1253. 1895 .

B Sachs. 1. c., p. 609. 
maintained as nearly as possible at the point of water saturation, uniformly moist sawdust, and fine-sieved garden earth. In some cases the primary roots were decapitated before the appearance of the secondary roots ( 5 centimeter roots were cut to I I or I 2 centimeters), in other cases the main roots were not decapitated. In any single experiment, however, all the seedlings were treated alike. Compact sawdust was not employed as in the case of experiments with primary
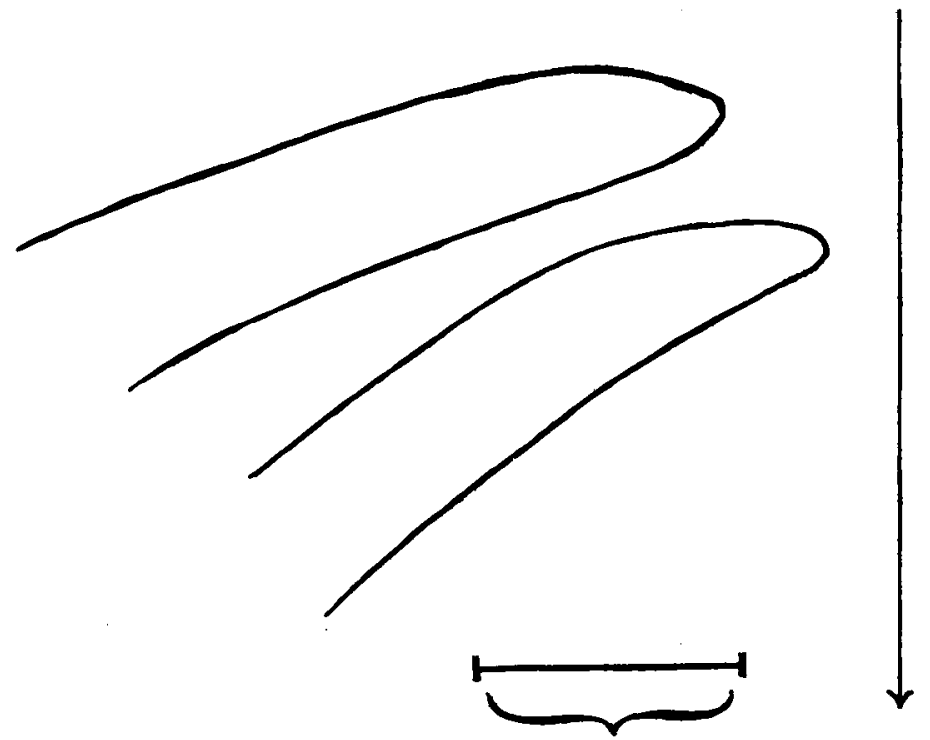

$1 \mathrm{~mm}$.

Fig. 2. Two secondary roots of Vicia faba var. equina which arose from a primary root planted horizontally in loose moist sawdust and grew obliquely upward through that medium, maintaining a curvature of the extreme tip.

roots because the secondary roots always followed a very sinuous course in that medium as did also the slender primary roots of Vicia sativa L., Ervum lens and other species. This was no doubt due to the mechanical weakness of the roots and the resistance of the particles of the sawdust (which were considerably larger than those of the soil used) to the advance of the root tip. In loose sawdust and in soil, neither the very irregular curvatures which took place in compact sawdust nor the slight "wellenförmig" curvatures which Sachs ${ }^{6}$ observed in

Sachs, l. c. p. 6 II. 
soil cultures made their appearance. In view of the change of geotonus of secondary roots which Sachs, ${ }^{7} \mathrm{Stah}^{8}$ and $\mathrm{Czapek}^{9}$ have reported as accompanying temperature changes, all the cultures in a single experiment were kept at the same temperature. During the course of all the experiments the temperature varied from $15^{\circ}$ to $19^{\circ} \mathrm{C}$. The cultures were kept in darkness except for short periods during which observations, drawings or photographs were being made, because, as Czapek ${ }^{10}$ has shown, the geotonus of the secondary root changes when the root is illuminated. All cultures employed in a given experiment were exposed to light for the same length of time when the behavior of the roots was recorded.

When secondary roots growing in air were so displaced that they formed a greater angle with the normal direction of the main root than their limiting angle, a curvature followed which, as Sachs has stated, involved the whole growing region. I also found, in accordance with Sachs's observations, that this curvature was subsequently considerably flattened and thereafter further permanent curvature was very slight or entirely lacking unless the root was still far removed from its limiting angle. The roots, however, maintained after the flattening of the first curvature a curvature or strong asymmetry of the terminal $\mathrm{I}$ to $\mathrm{I} 1 / 2 \mathrm{~mm}$. The retention of this curvature or asymmetry of the tip was clearly dependent upon the maintenance of the turgor of the cells of the tip, for it could be caused to disappear and reappear repeatedly within a few minutes by alternately exposing the root to relatively dry air and spraying it with water. This curvature of the tip which is maintained by the root, although there may be scarcely any or indeed no permanent curvature, is shown in figure 2. It is similar to the curvature of the tip of the primary root which Nermec ${ }^{11}$ first reported. As in the case of the primary root, this curvature of the extreme tip of the secondary root does not pass over as a permanent curvature to the region behind the tip, but is continually being flattened and at the same time is being renewed by the cells of the growing point and the root cap.

${ }^{7}$ Sachs, 1. c. p. 624.

${ }^{8}$ Stahl, Einfluss des Lichtes auf den Geotropismus einiger Pflanzenorgane. Bericht. Deutsch. Bot. Ges. 2: 396.1880.

${ }^{9}$ Czapek, 1. c. p. 125 I.

${ }^{10}$ Czapek, 1. c. p. 1250.

"Nĕmec, Ueber die Wahrnehmung des Schwerkraftreizes bei den Pflanzen, Jahrb. Wiss. Bot. 36: 93ff. 1901. 
In water the behavior of the secondary root was, in my experiments, very much the same as in moist air, although growth persisted much longer than in air and as a result the roots soon became so long that they bent downward under their own weight. There was only slight active and permanent curvature of the roots in water except when they formed a very large angle with the normal position of rest.

In media offering resistance to the advance of the root, the behavior of the secondary root was directly parallel to that of the primary root. Roots which had grown for a time in the limiting angle in loose moist
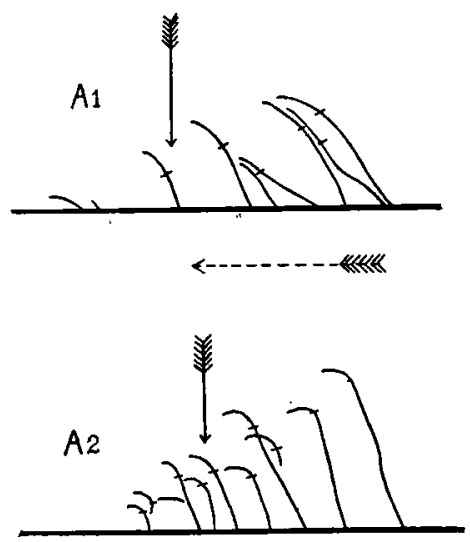
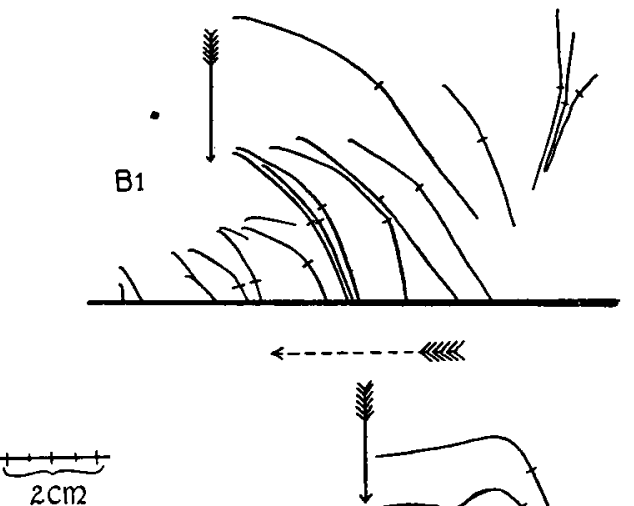

$\mathrm{B} 2$

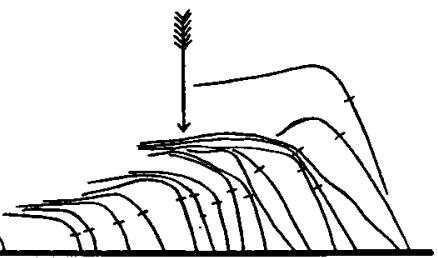

FIG. 3. Curvatures in loose moist sawdust and in earth of the secondary roots of Vicia $f a b a$ when displaced $90^{\circ}$ from the normal position. $A \mathbf{I}$ and $B \mathbf{I}$ represent roots growing in loose sawdust; $A 2$ and $B 2$, roots growing in earth. The arrows with dotted shaft show the direction of gravity relative to the root system at the time the secondary roots were of the length indicated by the cross strokes on the roots. It was at that time the cultures were turned through $90^{\circ}$. The arrows with solid shaft indicate the direction of gravity after the cultures were turned. Roots below the main root are omitted. Roots in $A$ I and $A 2$ grew for the same length of time in both positions; also roots in $B I$ and $B 2$.

sawdust curved very gradually toward the normal position when displaced upward from the original position, whereas roots growing in earth and similarly treated curved promptly and acutely into a position approximately the same as that from which they were displaced. This difference in behavior is represented in figure 3 , which was traced 
from photographs of roots in earth and loose moist sawdust cultures. Within a few hours after they had been displaced from their limiting angle, the roots in both media showed a very distinct curvature of the tip which was more distinct in the case of roots in loose sawdust than in the case of those in earth. As the roots in the former medium continued to grow, the curvature of the tip was continually flattened behind and was constantly reformed at the very extremity of the root, as in the case of roots in air. The flattening was not, however, complete and there remained a slight permanent curvature. In some cases this permanent curvature was so slight that it was perceptible only after the root had elongated three or four centimeters. The curvature of the tips of roots in soil was almost completely fixed and as the roots elongated an acute permanent curvature resulted which brought the root tip into a position not much $\left(5^{\circ}-20^{\circ}\right)$ removed from the former limiting angle. ${ }^{12}$ Thus a secondary root in earth generally reached within 24 hours at $15^{\circ}$ to $16^{\circ} \mathrm{C}$. a position which was attained by a root in loose sawdust only after 3 to 5 days. The secondary roots on the lower side of a horizontally placed main root often grew for 2 or 3 days in either of the media without showing any tendency to curve upward. This was due apparently to two factors. The first of these is the relatively slight angle these roots formed with the normal position of the secondary roots. The second is the still unexplained tendency of secondary roots which are displaced downward from their limiting angle to react less promptly and intensely than secondary roots which have been equally diverted above the normal position of rest. ${ }^{13}$ The downward directed secondary roots, however, curve more promptly upward when growing in earth than when they are in loose sawdust.

The very close parallel which exists between the behavior of secondary roots under stimulus of gravity in different media and primary roots under the same conditions indicates that the part played by the medium in both cases is the same. After flattening of the primary curvature mechanical resistance to change in the root's form and to the advance of the root is necessary to complete reaction and within certain limits the greater this resistance is the more promptly the reaction is completed. As in the case of the primary

12 Sachs (1. c. p. 627) noted the increase of the bounding angle following each curvature.

${ }^{13}$ Czapek, 1. c. p. $328 \mathrm{ff}$. 
roots, it is probably by hindrance of the flattening of the curvature of the root tip and by passive depression of the curved tip as it is pushed forward through the medium that the resistance of the medium reinforces and renders complete the geotropic reaction.

UNIVERSITY OF MiCHIGAN, ANN ARBor 